\title{
Aspectos moleculares e citotóxicos do paracetamol: uma revisão narrativa
}

\author{
Molecular and cytotoxic aspects of acetaminophen: a narrative review
}

Aspectos moleculares y citotóxicos del acetaminofén: una revisión narrativa

Manoel Thomáz de Farias $^{1 *}$, Carlos Humbertto Marques Cavalcanti ${ }^{1}$, José Rodrigo Alves Albuquerque $^{1}$, Álvaro Sérgio Soares Falcão Filho ${ }^{1}$, Paola Xavier Araújo ${ }^{1}$, Alanna Barbosa Mendonça Melo ${ }^{1}$, Robson de Jesus ${ }^{1}$.

\section{RESUMO}

Objetivo: Descrever os aspectos moleculares relacionados ao paracetamol e o mecanismo hepatotóxico desencadeado por doses elevadas. Revisão bibliográfica: A pesquisa realizada incluiu a absorção e a fase I da biotransformação do paracetamol, que resulta na formação do metabólito tóxico $\mathrm{N}$-acetil-pbenzoquinonaimina (NAPQI). A sobredose de paracetamol expõe o usuário à intoxicação aguda. Nesses casos de intoxicação, ocorre a depleção da glutationa, uma substância que participa do metabolismo do paracetamol convertendo o NAPQI em ácido mercaptúrico para posterior eliminação renal. Consequentemente, o metabólito tóxico acumula-se no organismo, provocando danos às mitocôndrias, deterioração de proteínas estruturais, podendo resultar na hepatotoxicidade. Nas situações de intoxicação, a substância N-acetilcisteína apresenta grande relevância clínica por contribuir para elevação da concentração da glutationa e redução da concentração da NAPQI acumulada. Considerações finais: Entende-se que o uso indiscriminado do paracetamol traz riscos consideráveis à saúde, incluindo desfechos fatais devido ao seu mecanismo hepatotóxico, sendo importante a instrução da população pelos profissionais de saúde acerca dessa problemática. Além disso, é fundamental reconhecer a importância do uso da N-acetilcisteína como antídoto em casos de intoxicação.

Palavras-chave: Paracetamol, Farmacocinética, Farmacodinâmica, Automedicação, Intoxicação.

\begin{abstract}
Objective: To describe the molecular aspects related to paracetamol and the hepatotoxic mechanism triggered by higher doses. Bibliographic review: The research performed included the absorption and phase I of biotransformation of paracetamol, which results in the formation of the toxic metabolite $\mathrm{N}$-acetyl-pbenzoquinoneimine (NAPQI). Acetaminophen overdose exposes the user to acute intoxication. In these cases of intoxication, glutathione is depleted, a substance that participates in the metabolism of paracetamol converting NAPQI into mercapturic acid for subsequent renal elimination. Consequently, the toxic metabolite accumulates in the body, causing damage to the mitochondrias, deterioration of structural proteins, which can result in hepatotoxicity. In situations of intoxication, the substance $\mathrm{N}$-acetylcysteine has great clinical relevance as it contributes to an increase in the concentration of glutathione and a reduction in the concentration of accumulated NAPQI. Final considerations: It is understood that the indiscriminate use of paracetamol leads to considerable health risks, including fatal outcomes due to its hepatotoxic mechanism, and it is important to educate the population by health professionals about this problem. Furthermore, it is essential to recognize the importance of using $\mathrm{N}$-acetylcysteine as an antidote in cases of intoxication.
\end{abstract}

Key words: Paracetamol, Pharmacokinetics, Pharmacodynamics, Self-Medication, Intoxication.

\footnotetext{
${ }^{1}$ Faculdade Ages de Medicina (FAM), Jacobina - BA.

*E-mail: manoelthfarias@hotmail.com
} 


\section{RESUMEN}

Objetivo: Describir aspectos moleculares relacionados con la toxicidad del paracetamol y el mecanismo hepatotóxico desencadenado por altas dosis. Revisión bibliográfica: La investigación realizada incluye la absorción y biotransformación del paracetamol, siendo este último producto de la fase biotransformativa I el metabolito tóxico $\mathrm{N}$-acetil-p benzoquinona imina (NAPQI). La sobredosis de acetaminofén expone al usuario a una intoxicación aguda. En estos casos de intoxicación se agota el glutatión, sustancia que participa en el metabolismo del paracetamol convirtiendo el NAPQI en ácido mercaptúrico para su posterior eliminación renal. En consecuencia, el metabolito tóxico se acumula en el cuerpo, causando daño a las mitocondrias, deterioro de las proteínas estructurales, lo que puede resultar en hepatotoxicidad. En situaciones de intoxicación, la sustancia $\mathrm{N}$-acetilcisteína tiene gran relevancia clínica ya que contribuye a un aumento de la concentración de glutatión y a una reducción de la concentración de NAPQI acumulado. Observaciones finales: Se entiende que el uso indiscriminado de paracetamol trae considerables riesgos para la salud, incluyendo desenlaces fatales debido a su mecanismo hepatotóxico, siendo importante que los profesionales de la salud eduquen a la población sobre este problema. Además, es fundamental reconocer la importancia de utilizar $\mathrm{N}$-acetilcisteína como antídoto en casos de intoxicación.

Palabras clave: Paracetamol, Farmacocinética, Farmacodinamia, Automedicación, Intoxicación.

\section{INTRODUÇÃO}

O paracetamol é um fármaco de ação antipirética e analgésica, mas com baixo mecanismo antiinflamatório. Sua produção e primeira utilização se deram, respectivamente, no ano de 1878 e 1887 . Vale ressaltar que, em 1950, através do pesquisador Bernard Beryl Brodie e do bioquímico Julius Axelrod, descobriu-se que o acetaminofeno, como também é denominado, se apresentava como um metabólito ativo da Fenacetina. Dessa forma, inicialmente, sua função foi a de assumir o posto desta, principalmente devido à nefrotoxicidade que a substância referida apresentava. Atualmente, suas indicações estão voltadas para condições como dor de cabeça, febre e resfriados (MOREIRA JRM, 2016; VIEIRA AL e FRANÇA GG, 2015).

Importante lembrar que a febre é abordada como a elevação da temperatura corpórea desencadeada por pirógenos, a exemplo das interleucinas, que atuam sobre o Órgão Vascular da Lâmina Terminal (OVLT), favorecendo o surgimento da Prostaglandina E2 (PGE2), que interage com a região hipotalâmica pré-óptica, dividida nos centros dissipador e promotor de calor. Analisando tal fato, o paracetamol, objetivando a redução térmica, inibe a síntese de prostaglandinas no Sistema Nervoso Central (SNC), além de reduzir a ligação do receptor N-metil-D-aspartato (NMDA) hipotalâmico com pirógenos (KUMAR V, et al., 2016); MÜHLBAUER M, 2016).

Já na dor, a ação deste fármaco é explicada por meio das cicloxigenases (COX's), que são vias pelas quais o ácido araquidônico, substância liberada da membrana fosfolipídica, mais especificamente por meio da enzima fosfolipase A2, é inserido e promove a geração de prostaglandinas, que são capazes de estimular os nociceptores. Dessa maneira, para reduzir o caráter doloroso, as COX's são bloqueadas (LIMA CP, et al., 2020; TONON AV, et al., 2020).

Além do descrito, o paracetamol é capaz de agir sobre os receptores $\mathrm{N}$-metil-D-aspartato medulares, apresentando mecanismo antagonista (CASTRO PLP, 2014). NMDA é a nomenclatura utilizada para designar receptores caracterizados por inotropismo, que possuem intrínseca relação com o glutamato, aminoácido de ação excitatória e que, ao estabelecer ligação com os receptores já referidos, apresenta importante associação com a dor, principalmente a crônica. Vale frisar que, em condições normais, o NMDA se encontra em um estado inativo promovido pelo magnésio (CHAW SH, et al., 2017; VALLI LG e SOBRINHO JA, 2014).

Além do exposto, vale acentuar que, com relação às doses de paracetamol adequadas, o limite máximo diário recomendado é de 4 gramas para adultos, enquanto, para crianças, a administração deve ser realizada com base na massa corporal, respeitando o limite máximo diário de 75 miligramas por quilo de peso corporal. 
Tais orientações são relevantes à medida que delimitam um espaço seguro para o não desenvolvimento de efeitos hepatotóxicos (MOREIRA JRM, 2016).

Diante de todo o contexto já inicialmente abordado, o presente estudo busca evidenciar os aspectos farmacológicos moleculares do paracetamol que, introduzidos a uma prática irrestrita, são observados como fatores predisponentes para distúrbios hepáticos.

\section{REVISÃO BIBLIOGRÁFICA}

\section{Conceitos gerais}

Medicamentos são definidos como substâncias destinadas a apresentar efeitos que variam desde uma terapêutica de prevenção e cura, até mesmo relacionados ao processo diagnóstico. Inicialmente, no século $\mathrm{XX}$, a base farmacológica era de origem natural, com formulações baseadas em prescrição e preparo artesanal. Através da evolução no campo farmacêutico, a produção passou a ocorrer em larga escala, o que foi fruto da veloz confecção de medicamentos e da intensa atividade comercial, ambas associadas ao avanço da economia mundial (GONÇALVES CA, et al., 2017).

Nos dias atuais, com a presença marcante de uma ferramenta publicitária irregular, da compra de muitos medicamentos sem a necessidade de receita e da manipulação incorreta, com doses superiores aos valores recomendados, uma intoxicação medicamentosa pode ser estabelecida, condição caracterizada por desenvolver um conjunto de sinais e sintomas que geralmente são específicos para o tipo de fármaco que 0 indivíduo entrou em contato (MOTA SF, et al., 2020; GONÇALVES CA, et al., 2017).

\section{Cenário nacional de intoxicação medicamentosa}

O processo de intoxicação é abordado como um problema de saúde pública devido ao severo impacto que desencadeia, tendo em vista o surgimento de novos casos e a grande quantidade de óbitos, gerando mais de 7.500 mil mortes em um período de 20 anos, de 1986 a 2006, e que, em um aspecto de origem, podem ser desencadeados, por exemplo, por situações acidentais e suicidas (OLIVEIRA JFM, et al., 2017).

O SINITOX, Sistema Nacional de Informações Toxicológicas, que atua na divulgação de dados associados à incidência anual de intoxicações no território nacional, evidencia que os fármacos são os mais relevantes promotores de casos envolvendo intoxicações desde a década de 90 , mais especificamente a partir do ano de 1994 (SILVA VT, et al., 2021). Atualmente, o Brasil pertence à classe de nações com as maiores taxas de consumo de medicamentos. Tal fato está intimamente ligado à automedicação, que representa, no país, cerca de $35 \%$ dos fármacos que são vendidos (SERENO VMB, et al., 2020).

Enquanto os adultos correspondem a cerca de 13, 94\% das intoxicações medicamentosas, a faixa etária infantil representa mais de $26 \%$, sendo as crianças com idade inferior a 5 anos as principais deste grupo. Ademais, é indispensável relatar que, durante o intervalo de tempo de 5 anos, de 2013 a 2017, a região sudeste foi responsável pelas maiores taxas de intoxicação no país, com 63.489 mil. Já o Norte, com os menores números, apresentou 1242 casos. O sexo feminino e o masculino tiveram, respectivamente, no mesmo espaço de anos, 63.648 e 36.843 casos. Entre os grupos farmacológicos que mais se relacionam com a intoxicação, se destacam os benzodiazepínico, analgésicos, antidepressivos e anticonvulsivantes (GONÇALVES CA, et al., 2017; SERENO VMB, et al., 2020).

O paracetamol, foco da discussão, é um fármaco bastante utilizado e que, associado à automedicação por apresentar característica de custo reduzido e alta acessibilidade, é determinante para condições que podem variar de não tão complexas, até muito graves, como o câncer de fígado (GLINKA C e RIBEIRO CNM, 2018).

\section{Farmacocinética do paracetamol}

A interação farmacológica do paracetamol com o organismo ocorre através de dois processos, conhecidos como farmacocinética e farmacodinâmica. Respectivamente, a primeira etapa envolve a administração, absorção, distribuição, biotransformação e eliminação, enquanto a segunda etapa engloba os mecanismos de ação estabelecidos pelo fármaco (RANG HP, et al., 2016). 
A administração geralmente ocorre através da via oral, em que há a possibilidade de aplicação do medicamento em forma de comprimido, cuja biodisponibilidade pode atingir níveis superiores a $60 \%$, podendo alcançar até $89 \%$, considerando o efeito de primeira passagem que abrange aproximadamente $20 \%$ da dose administrada. A aplicação do fármaco na forma farmacêutica líquida também se apresenta como uma possibilidade. O pico máximo de concentração na corrente sanguínea ocorre em até meia-hora depois da administração na forma líquida e entre 45 e 60 minutos quando administrada na forma de comprimido (CASTRO PLP, 2014; MEZAROBBA G e BITENCOURT RM, 2018).

O paracetamol também pode ser administrado pela via intravenosa, contribuindo para proporcionar maior biodisponibilidade e alcance de picos plasmáticos de $30 \mathrm{mg} / \mathrm{L} \mathrm{em}$ aproximadamente 15 minutos, após administração de $1 \mathrm{~g}$ da droga (CASTRO PLP, 2014).

Após a administração do paracetamol, através do sistema gastrointestinal, a droga é submetida à absorção e, antes de adentrar o sangue sistêmico, uma parcela do medicamento passa por metabolização hepática, processo conhecido como efeito de primeira passagem. Para ultrapassar a membrana celular, esse fármaco é difundido de forma passiva (CASTRO PLP, 2014). Vale ressaltar que a absorção está intimamente ligada com diversos processos, podendo estes, a depender, contribuir positivamente ou até inibi-la. São exemplos de possíveis interferentes: $\mathrm{pH}$, Glicoproteína $\mathrm{P}$, fluxo sanguíneo, sítio de absorção (região intestinal com presença de microvilosidades e borda em escova), entre outros (RANG HP, et al., 2016).

A distribuição do paracetamol se refere ao momento em que a substância farmacológica, após ser absorvida, deixa o sangue e vai em direção ao meio intracelular, atingindo antes, o interstício. Assim como a absorção, a distribuição depende de alguns fatores, são eles: débito cardíaco, associação do fármaco a proteínas plasmáticas, fluxo sanguíneo, grau de permeabilidade capilar, e aspecto químico, a exemplo da característica lipofílica (RANG HP, et al., 2016).

\section{Biotransformação do paracetamol}

O paracetamol passa por fases biotransformativas do tipo I e II no sítio hepático. A primeira, cuja mediação ocorre através do Citocromo P450, um sistema composto por enzimas, mais especificamente as monooxigenases, apresenta como produto do seu processo uma substância tóxica: $\mathrm{N}$-acetil-p-benzoquinonaimina, mais conhecida como NAPQI. Através da segunda etapa de biotransformação, a fase dois, o paracetamol se torna mais hidrossolúvel por meio da sulfatação e glucuronidação, que de maneira respectiva, são reações promovidas pelas sulfotransferases e UDP-Glucuronil-transferase e que, ainda de forma subsequente, possuem ações baseadas na transferências dos grupos sulfo e glucuronosil para o fármaco citado anteriormente, objetivando assim, gerar substâncias que são capazes de sofrer excreção urinária (MOREIRA JRM, 2016; JÚNIOR JGS, et al., 2019; GLINKA C e RIBEIRO CNM, 2018).

Em condições normais e na presença de valores considerados adequados, as taxas do NAPQI formado são baixas e, por meio da ligação deste com a região tiol da Glutationa, um agente que atua na captação de radicais livres, ocorre a formação de um produto que pode ser eliminado do organismo através do mecanismo renal, o ácido mercaptúrico (MOREIRA JRM, 2016).

\section{Glutationa: metabolismo e funções}

A glutationa (GSH) é um tripeptídeo linear de aminoácidos composto por L-gama-glutamil-L-cisteinilglicina. É sintetizada no interior das células (citosol) a partir da $\gamma$-glutamilcisteína sintetase (GCS) que utiliza o glutamato e a cisteína como substratos (dipeptídeo y -GluCys). Dessa forma, se une com a glicina pela ação da glutationa sintetase, dando origem a GSH. Está presente no organismo em três formas, reduzida (maior parte), oxidada e conjugada, atuando de forma direta e indireta em vários processos biológicos, destacandose a destoxificação e proteção celular. A síntese desta molécula, bem como seu metabolismo, é dependente da biodisponibilidade dos aminoácidos (principalmente a cisteína), onde, uma falha nestes processos se associa a diversas patologias (PAIXÃO JNV, 2018; NETO ASSR, 2010; JÚNIOR JGS, et al., 2019).

Levando em consideração a constante exposição e as características organizacionais de compostos químicos estranhos ao corpo humano (xenobióticos), onde os mesmos, em sua maioria, se ligam fortemente 
a proteínas, faz-se necessário a atuação de importantes mecanismos para a metabolização (hepática) e excreção (renal) destas substâncias. Esta metabolização visa, sobretudo, modificar a solubilidade destes compostos, tornando-os mais hidrossolúveis e menos reativos do que as moléculas originais (HUPER PC, et al., 2008; GONÇALVES ES, et al., 2014).

Considera-se que esse mecanismo se proceda em três fases, onde na I e II ocorrem as modificações nos xenobióticos e na fase III a eliminação para o meio extracelular com posterior excreção. Na fase I, Sob a ação de enzimas específicas, grupos químicos funcionais são introduzidos nas moléculas xenobióticas para facilitar sua eliminação, no entanto, nesta reação ocorre a liberação de agentes tóxicos aumentando a reatividade desses produtos resultantes (bioativação), o que é prontamente suplantado pelas reações da fase seguinte, explicando a necessidade de estas ocorrerem de forma concomitante. Já na fase II ocorre a conjugação destes metabólitos reativos (fase I) com outras moléculas polares que, por sua vez, vão atuar na inativação e eliminação destes compostos (HUBER PC, et al., 2008; GONÇALVES ES, et al., 2014).

$\mathrm{Na}$ função de defesa celular contra xenobióticos, a GSH atua de forma conjunta com a enzima glutationa - S - transferase (GST). Esta enzima está localizada em sua maioria no citoplasma das células hepáticas e vai atuar diretamente fornecendo transportadores para o conjugado glutationa/xenobióticos no intuito de facilitar sua eliminação, na maioria das vezes, de forma inalterada através da via biliar ou sendo convertido a ácido mercaptúrico para excreção renal como ocorre no metabolismo do paracetamol. Esta união (GSH/xenobiótico) representa de forma quantitativa a principal reação observada na fase II da destoxificação enzimática dos xenobióticos (HUBER PC, et al., 2008).

No meio extracelular, após o reconhecimento da ectoenzima y-glutamiltranspeptidase (GGT), ocorrem ciclos para a degradação e utilização da glutationa assim como dos conjugados, sendo que, o catabolismo de conjugados de glutationa leva à formação de conjugados com a cisteína (Cys-SR) e estes por sua vez poderão servir de substrato para novas moléculas. Nos espaços apicais e compartimentos sinusoidais ocorre um extenso catabolismo seguido de recaptação celular de alguns produtos oriundos da degradação. Posteriormente pode haver uma reutilização destes produtos ou uma conversão de S-conjugados de cisteína em ácido mercaptúrico que será exportado a partir das células para eliminação através da urina ou fezes (NETO ASSR, 2010).

\section{Mecanismo de lesão hepática}

Nos casos em que doses do paracetamol que excedem as faixas terapêuticas são ingeridas, a fase II da biotransformação se torna sobrecarregada, há depleção da glutationa, favorecendo o acúmulo da $\mathrm{N}$-acetil-p benzoquinona imina, dando início à hepatotoxicidade (MOREIRA JRM, 2016; TERRES DR, 2015; COSTA IAF e OLIVEIRA FS, 2021; CAIRES CRS, et al., 2018).

O NAPQI, agora livre e em grandes quantidades, pode estabelecer associação, por exemplo, com proteínas celulares, utilizando como base de recepção o grupo tiol dos aminoácidos destas últimas, sendo as proteínas das mitocôndrias focos especiais neste contexto. A mitocôndria, uma organela de extrema importância, à medida que é afetada pelos níveis elevados de NAPQI, apresenta distúrbios em seu funcionamento, levando à disfunção na respiração celular, redução do ATP e desencadeando a síntese de espécies reativas de oxigênio, favorecendo a formação do peroxinitrito, que se origina através do óxido nítrico, uma das espécies liberadas no contexto trabalhado, com o ânion superóxido e é capaz de promover lesões no DNA da organela (FREITAS JAB, et al., 2017; MOREIRA JRM, 2016).

O estresse oxidativo baseado no Peroxinitrito e em outras espécies reativas produzidas, associado ao esgotamento da glutationa, torna a membrana da mitocôndria mais permeável. Isso favorece a saída do Citocromo $C$ por causa da ativação e combinação das proteínas BAK e BAX. A caspase-9, que é acionada pelo citocromo abordado, reage com o fator ativador de apoptose, originando o apoptossomo e propiciando a ativação de outras categorias de caspases e do mecanismo de apoptose da célula. Além disso, devido à instabilidade das concentrações de cálcio no interior mitocondrial e no interior celular, estando em maiores quantidades no citosol, as proteínas estruturais podem ser deterioradas e o hepatócito entrar em necrose (CASTRO PLP, 2014). 
Outra via de distúrbio hepático oriundo do uso indiscriminado do paracetamol envolve o necroptosoma, um sistema de proteínas no qual se encontram receptores de interação serina/treonina-proteína quinase do tipo 1, conhecidos como RIPK-1, que promovem hepatotoxicidade e estão relacionados com o processo de morte celular (MOREIRA JRM, 2016).

Em suma, faz-se necessário ressaltar que as manifestações clínicas da toxicidade desencadeada pelo paracetamol variam conforme o tempo. Assim, podem ser citadas: Fase 1: Ausência de Injúria Hepática, primeiras 24 horas (pacientes assintomáticos e manifestações clínicas inespecíficas, como náuseas e vômitos), Fase 2: Início da Injúria Hepática, de 24 a 72horas (dor no quadrante superior direito e elevação da Aspartato Aminotransferase), Fase 3: Hepatotoxicidade Máxima, de 72 a 96 horas (Reaparecimento dos sintomas da primeira fase simultaneamente a icterícia, confusão mental - decorrente da encefalopatia hepática, além de coma e hemorragia) e Fase 4: Resolução, de 4 dias a 2 semanas (Indivíduos que resistiram à terceira etapa são submetidos a um estágio de restabelecimento hepático, especialmente da função do órgão. A reabilitação histológica transcorre após o avanço clínico, havendo possibilidade de um período de demora de até três meses) (WHALEN K, et al., 2016).

\section{Antídoto: a função da $\mathrm{N}$-acetilcisteína em casos de intoxicação}

O uso de $\mathrm{N}$-acetilcisteína em casos de intoxicação por acetaminofeno ocorreu há 50 anos atrás, na década de 70, sendo a Inglaterra o país origem do seu emprego. Inicialmente, visando analisar a eficácia do antídoto para evitar o desencadeamento de efeitos negativos, como a toxicidade hepática, animais foram submetidos a experiências, contexto que foi, anos depois, procedido por um ensaio clínico randomizado, dessa vez, em seres humanos, mais especificamente aqueles que apresentavam insuficiência hepática produzida pelo paracetamol, objetivando evidenciar a sua aplicabilidade nessas situações (JÚNIOR JGS, et al., 2019).

Importante destacar que um dos mais importantes estudos envolvendo a N-acetilcisteína (NAC), de caráter multicêntrico, sucedido entre 1976 e 1985, demonstrou que indivíduos que haviam ingerido o paracetamol e foram expostos ao tratamento com NAC em uma faixa de tempo variando de 8 a 10 horas após, tiveram menos riscos de apresentarem hepatotoxicidade quando contrapostos aos que receberam a terapêutica 10 horas a 1 dia depois de terem entrado em contato com o fármaco (JÚNIOR JGS, et al., 2019).

A obtenção deste medicamento (NAC) se dá a partir do aminoácido L-cisteína e sua aplicabilidade se relaciona com a capacidade que possui como antídoto em casos de intoxicação aguda por paracetamol. Tratase de uma substância que contém grupo sulfidrila, aos quais os metabólitos tóxicos podem se fixar, promovendo destoxificação do sistema hepático. Ademais, a NAC atua inicialmente como precursor substituto da glutationa e auxilia as reações de sulfatação. Além disso, esse antídoto pode funcionar como um antioxidante e ajudar na recuperação (WHALEN K, et al., 2016).

Em síntese, a N-acetilcisteína (NAC) repõe os estoques de glutationa hepática, aumentando a conjugação desta substância ao NAPQI e sua consequente destoxificação. Vale ressaltar que sua administração deve ser estabelecida em qualquer paciente com possibilidades de desenvolvimento de lesão hepática, ou seja, com taxas sanguíneas de acetaminofeno superiores à linha de possível toxicidade no nomograma de RumackMatthew ou história de ingestão de doses tóxicas e concentração e tempo de ingestão que não puderam ser delimitados (FREITAS JAB, et al., 2017; WHALEN K, et al., 2016).

\section{CONSIDERAÇÕES FINAIS}

A revisão em questão buscou englobar os diversos aspectos moleculares e tóxicos que permeiam o uso em demasia do paracetamol, um analgésico e antipirético, mas com propriedades anti-inflamatórias periféricas mínimas, sendo o mais utilizado no mundo. Salienta-se que um dos fatores que mais contribuem para o problema de saúde pública que o paracetamol representa é a automedicação. $O$ baixo custo, a alta acessibilidade e a sobredose tornam o uso desse fármaco um problema de saúde, estando estes relacionados a eventos como hepatotoxicidade e condições mais complexas. Tal contexto, de forma indireta, demonstra a relevância científica que o debate atual representa ao ponto em que retrata os riscos de uma prática que está 
enraizada na rotina de muitos indivíduos. Por fim, destaca-se que mesmo havendo uma ampla abordagem sobre o paracetamol, algumas informações importantes pertenciam a reservatórios anteriores aos últimos dez anos.

\section{REFERÊNCIAS}

1. CAIRES CRS, et al. Intoxicação medicamentosa com foco nos efeitos do paracetamol. Revista Científica, 2018; 1(1): $1-11$.

2. CASTRO PLP. Farmacocinética do paracetamol. Dissertação (Mestrado em Ciências Farmacêuticas) - Faculdade de Ciências da Saúde. Universidade Fernando Pessoa, Porto, 2014; 97p.

3. CHAW SH, et al. Anestesia em encefalite antirreceptor de N-metil-D-aspartato-a anestesia geral é um requisito? Relato de caso. Revista Brasileira de Anestesiologia, 2017; 67(6): 647-650.

4. COSTA IAF, OLIVEIRA FS. Fármacos Hepatotóxicos e Hepatoprotetores: uma revisão de literatura. Journal of Biology \& Pharmacy and Agricultural Management, 2021; 1(17): 143-164.

5. FREITAS JAB, et al. Medicamentos isentos de prescrição: perfil de consumo e os riscos tóxicos do paracetamol. Revinter, 2017; 10(3): 134-154.

6. FREITAS KB, et al. Uso indiscriminado do paracetamol no Rio Grande do Sul: perfil de uma década. Saúde e Desenvolvimento Humano, 2020; 8(2): 45-53.

7. GLINKA C, RIBEIRO CNM. O Papel do Paracetamol na Hepatite Medicamentosa. Revista Eletrônica Biociências, Biotecnologia e Saúde, 2018; 21: 46-54.

8. GONÇALVES CA, et al. Intoxicação medicamentosa: relacionada ao uso indiscriminado de medicamentos. Revista Científica da Faculdade de Educação e Meio Ambiente, 2017; 8(1): 135-143.

9. GONÇALVES ES, et al. A importância da determinação analítica de intermediários reativos e de seus produtos de reações com biomacromoléculas: uma mini revisão. Química nova, 2014; 37: 317-322.

10. HANG HP, et al. Hang \& Dale: Farmacologia. 8nd ed. Rio de Janeiro: Elsevier, 2016; 1939 p.

11. HUBER PC, et al. Glutationa e enzimas relacionadas: papel biológico e importância em processos patológicos. Química Nova, 2008; 31(5): 1170-1179.

12. JÚNIOR JGS, et al. Hepatotoxicidade induzida pelo paracetamol e a utilização do nomograma de Rumack-Matthew para avaliar a terapêutica com n-acetilcisteína. Revista Uningá, 2019; 56(4): 65-84.

13. KUMAR V, et al. Robbins \& Cotran Patologia - Bases Patológicas das Doenças. 9nd ed. Rio de Janeiro: Elsevier, 2016; 1480p.

14. LIMA CP, et al. Avaliação farmacêutica dos riscos do uso dos anti-inflamatórios não esteroidais. Unisanta Health Science, 2020; 4(1): 1-20.

15. LORENZONI AA, et al. Efeito protetor de produtos naturais sobre o dano hepático induzido pelo paracetamol. Revista Acta Ambiental Catarinense, 2014; 11(1/2): 43-52.

16. MEZAROBBA G, BITENCOURT RM. Toxicidade do paracetamol: o álcool como um fator de risco. Unoesc \& CiênciaACBS, 2018; 9(1): 105-112.

17. MOREIRA JRM. Intoxicações por Paracetamol: Metabolismo, Mecanismos de Toxicidade e Novas Abordagens da Terapêutica. Dissertação (Mestrado Integrado em Ciências Farmacêuticas) - Universidade de Coimbra. Faculdade de Farmácia da Universidade de Coimbra, Coimbra, 2016; 33p.

18. MOTA SF, et al. Caracterização do perfil das intoxicações medicamentosas na população de Taubaté, São Paulo, no período de 2014 a 2018. Brazilian Journal of Health Review, 2020; 3(5): 12672-12683.

19. MÜHLBAUER M. Paracetamol, um AINE particular. Ciência Atual-Revista Científica Multidisciplinar do Centro Universitário São José, 2016; 7(1): 1-10.

20. NETO ASSR. Glutationa: envolvimento em defesa antioxidante, regulação de morte celular programada e destoxificação de drogas. Dissertação (Mestrado em Ciências Farmacêuticas) - Faculdade Ciências da Saúde. Universidade Fernando Pessoa, Porto, 2010; 77 p.

21. OLIVEIRA JFM, et al. Tendência da mortalidade por intoxicação medicamentosa entre gêneros e faixas etárias no Estado de São Paulo, Brasil, 1996-2012. Ciência \& Saúde Coletiva, 2017; 22: 3381-3391.

22. OLIVEIRA MMC, et al. O uso crônico de anti-inflamatórios não-esteroidais e seus efeitos adversos. Revista Caderno de Medicina, 2019; 2(2): 90-100.

23. PAIXÃO JNV. Estudo de metabolismo da Saccharomyces cerevisiae para produção de glutationa utilizando melaço de beterraba. Dissertação (Mestrado em Engenharia Química) - Instituto de Química. Universidade Federal de Goiás, Goiânia, 2018; 102 p.

24. SERENO VMB, et al. Perfil epidemiológico das intoxicações por medicamentos no Brasil entre os anos de 2013 a 2017. Brazilian Journal of Development, 2020; 6(6): 33892-33903.

25. SILVA JCS, et al. A incidência do uso indiscriminado de medicamentos. Revista Brasileira Interdisciplinar de Saúde, 2020; 2(1): 95-99.

26. SILVA VT, et al. Intoxicação por medicamentos: uma revisão de literatura com abordagem no tratamento. Revista Eletrônica Acervo Científico, 2021; 23: e6781.

27. TERRES DR. Potencial toxicológico de medicamento de venda livre: ênfase no paracetamol. FACIDER-Revista Científica, 2016; (8): 1-15.

28. TONON AV, et al. Consequências da automedicação e uso indiscriminado do anti-inflamatório não esteróide paracetamol em adultos. Revista Artigos.Com, 2020; 22: e5797.

29. VALLI LG. Mecanismo de ação do glutamato no sistema nervoso central e a relação com doenças neurodegenerativas. Revista Brasileira de Neurologia e Psiquiatria, 2014; 18(1): 58-67.

30. VIEIRA AL, FRANÇA GG. As Consequências no Consumo Indiscriminado do Paracetamol e Orientação Farmacêutica à Promoção ao Uso Racional. Revista Acadêmica Oswaldo Cruz, 2015; 6(6): 1-12.

31. WHALEN K, et al. Farmacologia ilustrada. 6nd ed. Porto Alegre: Artmed, 2016; 680 p. 